\title{
Determinanten traditionell-sexistischer Einstellungen in Deutschland - eine Analyse mit Allbus-Daten
}

\author{
Anja Mays
}

Zusammenfassung: In der Bundesrepublik verfügt nach wie vor ein relevanter Teil der männlichen wie weiblichen Bevölkerung über traditionell-sexistische Rollenvorstellungen. Um ein besseres Verständnis in die Mechanismen ihrer Entwicklung zu bekommen, untersucht der vorliegende Beitrag Ausmaß und Bestimmungsgründe von Geschlechtsrollenvorstellungen. Dazu werden unter Verwendung der ALLBUS 2008-Daten Strukturgleichungsmodelle berechnet, die eine Differenzierung zwischen direkten und indirekten Einflussfaktoren erlauben. Es zeigt sich, dass für sexistische Einstellungen sowohl Effekte der Sozialisation als auch Einflüsse der Situation von Bedeutung sind. $\mathrm{Zu}$ den insgesamt wichtigsten Determinanten gehören bei beiden Geschlechtern: (mangelnde) Bildung, westdeutsche Herkunft, Geburt vor 1949, antiegalitäre Werthaltungen sowie, als bislang nicht untersuchte Erklärungsgröße, anomische Empfindungen. Zwei Effekte sind überraschenderweise ausschließlich beim männlichen Teil der Bevölkerung zu beobachten: Mütterliche Nicht-Berufstätigkeit sowie das Gefühl ökonomischer Deprivation führt nur bei Männern zu verstärkt traditionell-sexistischen Rollenvorstellungen. Aus diesen Befunden ergeben sich Ansatzpunkte für eine Reduktion sexistischer Geschlechtsrollenorientierungen.

Schlüsselwörter: Geschlecht · Geschlechtsrollen · Traditioneller Sexismus · Sozialisation · Antiegalitäre Wertorientierungen · Anomia $\cdot$ Strukturgleichungsmodelle

\section{Prevalence and determinants of traditional sexist attitudes in Germany-an analysis based on Allbus data}

\begin{abstract}
Traditional sexism is still often present in Germans of both genders. To elucidate the mechanism of its development the paper is seeking for determinants of gender roles. Based on the ALLBUS 2008 data set structural equation models were calculated to separate direct from indirect factors. Both, socialization as well as the situation, play a role in development of traditional sexism. The following determinants where found as most relevant in both genders: (insufficient) education, origin from the western part of Germany, birth earlier than 1949, anti-egalitarian values and, a factor previously not investigated, anomia. Surprisingly two other determinants of sexism were only seen in men: Not working mother and the feeling of economic deprivation. These results provide starting points to reduce sexist attitudes in the future.
\end{abstract}

C Die Autor(en) 2012. Dieser Artikel ist auf Springerlink.com mit Open Access verfügbar.

\footnotetext{
A. Mays $(\bowtie)$

Georg-August-Universität Göttingen, Wilhelmsplatz 1, 37073 Göttingen, Deutschland

E-Mail: anja.mays@sowi.uni-goettingen.de
} 
Keywords: Gender · Gender roles · Traditional sexism · Socialization · Antiegalitarian values · Anomia $\cdot$ Structuralequation models

\section{Einleitung}

Gleichstellung von Männern und Frauen ist bei uns nach wie vor nicht selbstverständlich. Zwar können Frauen mittlerweile Kanzlerin oder Bischöfin werden und nicht selten erreichen Frauen bessere Schul- und Universitätsabschlüsse als Männer. Im internationalen Vergleich fällt die Bundesrepublik jedoch in Sachen Gleichstellung zurück. Nach dem kürzlich erschienenen Ländervergleich des Weltwirtschaftsforums „Global Gender Gap Index 2010“ verliert Deutschland zwischen 2006 und 2010 sieben Plätze und belegt aktuell nur noch den 13. Rang (World Economic Forum 2010). Die größten Geschlechterunterschiede bestehen demnach in den Bereichen Gehälter und Karriereoptionen. So ist in der Bundesrepublik das Einkommensverhältnis zwischen Männern und Frauen mit einem female-to-male ratio - Wert von 0,64 deutlich zuungunsten der Frauen verschoben. ${ }^{1}$ Ebenfalls weit abgeschlagen sind Frauen in Führungspositionen: lediglich 5\% der 600 deutschen Großunternehmen wurden im Jahr 2009 von einer weiblichen Führungskraft geleitet (World Economic Forum 2010). Die Ungleichbehandlung von Frauen beschränkt sich jedoch nicht auf das Berufsleben. Während Frauen, selbst bei voller Berufstätigkeit, die Hauptlast der Haus- und Familienarbeit tragen, finden Männer für ihre oft nur geringe Beteiligung an der häuslichen Arbeit in ihren Partnerschaften mehr Anerkennung als Frauen (Vorwerk Familienstudie 2009). Die ungleiche Geschlechterbehandlung beginnt zudem nicht erst im Erwachsenenalter. Schon in der Kindheit werden Jungen ökonomisch bevorzugt und erhalten erheblich mehr Taschengeld als gleichaltrige Mädchen (z. B. über $30 \%$ mehr bei Viertklässlern, LBS-Kinderbarometer 2009). Darüber hinaus werden sie deutlich seltener für Arbeiten im elterlichen Haushalt herangezogen (Vorwerk Familienstudie 2009).

Die Tatsache, dass auch heute noch derartige Geschlechterungleichheiten sichtbar sind, deutet darauf hin, dass traditionelle Rollenmuster nach wie vor in der Gesellschaft verankert sind. Nur wenn ein relevanter Teil der Bevölkerung patriarchale gesellschaftliche Strukturen unterstützt, ist zu erwarten, dass geschlechtsspezifische Macht- und Statusunterschiede in der Gesellschaft dauerhaft bestehen bleiben (vgl. Endrikat 2003).

An diesem Aspekt knüpft der vorliegende Aufsatz an. Zum einen wird unter Verwendung der ALLBUS 2008-Daten untersucht, in welchem Ausmaß traditionell-sexistische Rollenvorstellungen, die Ungleichbehandlungen der Geschlechter legitimieren, in der Bevölkerung Unterstützung finden. Zum anderen geht es um die Identifizierung wichtiger Determinanten sexistischer Einstellungen. Da nur wenige Studien existieren, welche die Mechanismen der Einstellungsgenese im Detail untersuchen, versucht der vorliegende Artikel mit Hilfe von Pfadmodellen einen Beitrag zur Aufdeckung der Kausalstruktur

1 Ein Wert=1 bedeutet Geschlechtergleichstellung, Werte unter 1 benachteiligen Frauen, Werte über 1 benachteiligen Männer (World Economic Forum 2010). 
traditionell-sexistischer Einstellungen zu leisten. ${ }^{2}$ Dies geschieht, indem die Bestimmungsgründe in direkt und indirekt wirksame Faktoren zu zerlegt werden und somit eine detailliertere Untersuchung der Beziehungsstruktur der Determinanten möglich wird.

\section{Problemstellung}

Unter Sexismus können allgemein ,geschlechtsbezogene Stereotype, Affekte und Verhaltensweisen...“ verstanden werden, „...die einen ungleichen sozialen Status von Frauen und Männern zur Folge haben“ (Eckes 2008, S. 170). ${ }^{3}$ Die klassische Form des Sexismus ${ }^{4}$ umfasst traditionelle Rollenvorstellungen, die der Frau die Rolle der Ehefrau, Mutter und Karrierehelferin für den Mann zuteilt. Der Frau wird der Platz am heimischen Herd und in der privaten Sphäre zugewiesen, während der Mann die Ernährerrolle übernimmt und sich im öffentlichen Raum bewegt. Sätze wie „,ür eine Frau ist es wichtiger, ihrem Mann bei seiner Karriere zu helfen, als selbst Karriere zu machen“ oder „es ist für alle Beteiligten viel besser, wenn der Mann voll im Berufsleben steht und die Frau zu Hause bleibt und sich um den Haushalt und die Kinder kümmert" (ALLBUS-Items) stehen exemplarisch für solche traditionell-sexistischen Einstellungen. ${ }^{5}$ Diese Rollenauffassung impliziert aus mehreren Gründen eine Abwertung oder Benachteiligung von Frauen. Einerseits erfährt Haus- und Familienarbeit weniger gesellschaftliche Anerkennung als die vom Mann ausgeübte Berufstätigkeit und wird in der Regel unentgeltlich verrichtet. Dies führt meist $\mathrm{zu}$ wirtschaftlicher Abhängigkeit und hat oft Altersarmut von Frauen zur Folge. Andererseits wird Frauen, durch den Verweis auf den familiär-privaten Bereich, Entfaltungsmöglichkeit außerhalb der häuslichen Umgebung verweigert. Auf der strukturellen Ebene befördert die öffentliche Unsichtbarkeit von Frauen deren politische Machtlosigkeit und

2 Analysen, die sich mit den generellen Determinanten sexistischer Einstellungen beschäftigen, liegen für Deutschland vergleichsweise selten vor (einige wenige Beispiele z.B. Braun et al. 1994 oder Endrikat 2003). Die internationale und insbesondere die US-amerikanische Forschung kann in dieser Frage dagegen auf eine recht umfangreiche Forschungstradition zurückblicken (vgl. z. B.Tomeh 1979; Morgan und Walker 1983; Thornton et al. 1983; Banaszak und Plutzer 1993; Rhodebeck 1996; Bolzendahl und Myers 2004; Lee et al. 2007). Allerdings existieren auch hier nur wenige Studien, welche die Mechanismen der Einstellungsgenese im Detail untersuchen.

3 Der Begriff Sexismus kennzeichnet somit den Sachverhalt, dass Menschen aufgrund ihres Geschlechtes diskriminiert werden. Historisch entstand der Begriff des Sexismus in den 1960er Jahren in Anlehnung an das Konzept des Rassismus, der Menschen nach physischen Merkmalen wie der Hautfarbe diskriminiert (vgl. Räthzel 2004; Endrikat 2003; Swim et al. 1995).

4 Neben dem klassischen oder traditionellen Sexismus unterscheidet die Forschung mit den Konzepten des modernen und ambivalenten Sexismus zwei weitere Formen sexistischer Einstellungen (vgl. Eckes 2008; Six-Materna 2008; Glick und Fiske 2009). Da das vorliegende Datenmaterial keine Items zur Messung des modernen oder des ambivalenten Sexismus enthält, wird auf diese beiden Konzepte nicht näher eingegangen.

5 Synonym für den Begriff der sexistischen Einstellungen werden die Vokabeln „traditionelle“, „,antiegalitäre“ oder „patriachale“ Geschlechtsrollenvorstellungen benutzt. 
führt zu einer Konservierung der Status- und Machtunterschiede zwischen Männern und Frauen.

\subsection{Ausmaß sexistischer Einstellungen in Deutschland}

Untersuchungen, die sich mit der langfristigen Entwicklung geschlechtsbezogener Einstellungen in westlichen Industriestaaten befassen, zeigen, dass die offene oder traditionelle Form des Sexismus in den letzten Dekaden an Zustimmung verloren hat (Brewstwer und Padavic 2000; Bolzendahl und Myers 2004). Zurückgeführt wird dieser Einstellungswandel zumeist auf Perioden- und Kohorteneffekte (vgl. Braun et al. 1994; Brewstwer und Padavic 2000; Bolzendahl und Myers 2004; Lee et al. 2007). Auch für Deutschland ist ein deutlicher Einstellungswandel hin zu stärker egalitären Rollenvorstellungen sichtbar (Braun et al. 1994; Endrikat 2003; Hofäcker und Lück 2004; Kurz 2004; Lee et al. 2007). Gleichwohl besteht auch heute noch, insbesondere in den westlichen Landesteilen, ein z. T. bemerkenswertes Ausmaß an sexistischen Einstellungen.

Betrachtet man die drei klassischen Sexismus-Items im ALLBUS-Datensatz (2008) ${ }^{6}$, so schwanken die jeweils höchsten Zustimmungsraten, je nach Item, zwischen $28 \%$ und knapp über $41 \%$ (s. Abb. 1 und 3).

Die stärkste Unterstützung erreicht die Aussage, es sei für alle Beteiligten besser, wenn der Mann im Berufsleben stehe und sich die Frau um Familie und Haushalt kümmere; über $41 \%$ der westdeutschen Männer, aber auch über 39\% der westdeutschen Frauen vertreten diese Auffassung (Abb.1). Die ostdeutsche Bevölkerung, Männer wie Frauen, nimmt hier, wie auch bei den anderen Items, eine erheblich liberalere Haltung ein. Nur 19,1 \% Männer und 16,7\% Frauen stimmen diesem Statement zu.

Vergleicht man die verschiedenen Items miteinander, so zeigt sich ein einheitliches Muster der Zustimmung: Westdeutsche Männer sind, bis auf eine Ausnahme (Abb. 2), die Vorreiter traditioneller Auffassungen, dicht gefolgt von westdeutschen Frauen. Männer aus den ostdeutschen Bundesländern hingegen verfügen in allen drei Fällen über deutlich weniger traditionelle Geschlechtsrollenorientierungen als westdeutsche Frauen und Männer. Ostdeutsche Frauen schließlich lassen mit Zustimmungsraten von $13 \%$ bis $23 \%$ die geringsten traditionell-sexistischen Einstellungen erkennen (Abb. 1-3).

Vergleicht man die Einstellungsverteilung zwischen den Geschlechtern, so fällt auf, dass klassisch-sexistische Vorstellungen bei Frauen insgesamt fast ebenso oft anzutreffen sind wie bei Männern (ähnliche Befunde bei Endrikat 2003; Bolzendahl und Myers 2004; Lee et al. 2007). Endrikat versucht dieses Phänomen damit zu erklären, dass traditionelle Rollenvorstellungen bei einigen Frauen als mögliche Strategie gewählt werden, um ein positives Selbstbild zu erhalten („Ich will so leben, wie ich leben muss“, Endrikat 2003, S. 132).

Die unterschiedlichen Geschlechtsrollenorientierungen in Ost und West werden in der Literatur auf verschiedene Ursachen zurückgeführt. Zum einen wird argumentiert, die egalitär ausgerichtete Staatsideologie der DDR habe zur Ausprägung liberaler Geschlechtsrollenvorstellungen geführt (vgl. Endrikat 2003, S. 127; Westle 2009). Zum

6 Die in diesem Beitrag benutzten Daten entstammen der „Allgemeinen Bevölkerungsumfrage der Sozialwissenschaften“(Allbus 2008, Nr. 4600). 
Abb. 1: Sexismus - Item 1 (Quelle: ALLBUS 2008, eigene Berechnungen.)
"Es ist für alle Beteiligten viel besser, wenn der Mann voll im Berufsleben steht und die Frau zu Hause bleibt und sich um den Haushalt und die Kinder kümmert", Zustimmung in \%*

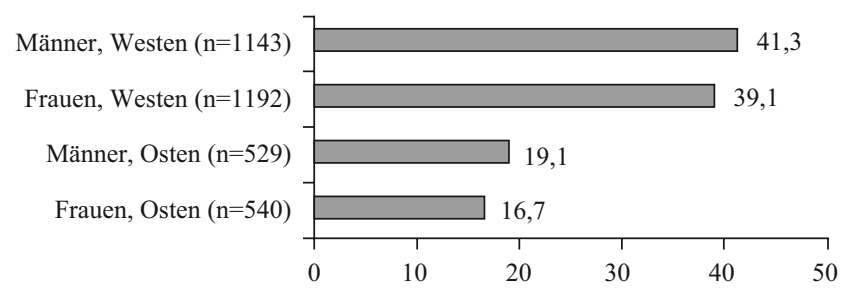

*Die Kategorien „stimme voll zu“ und „stimme eher zu“ wurden hier zusammengefasst.

\section{"Für eine Frau ist es wichtiger, ihrem Mann bei seiner Karriere zu helfen, als selbst Karriere zu machen”, Zustimmung in \%*} eigene Berechnungen.)

Abb. 3: Sexismus - Item 3 (Quelle: ALLBUS 2008, eigene Berechnungen.)

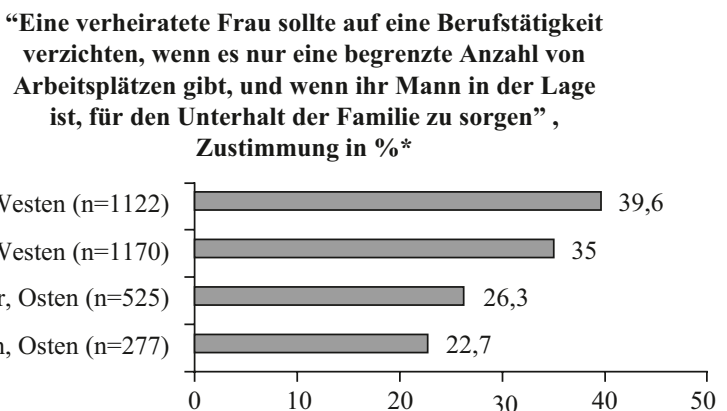

*Die Kategorien „stimme voll zu“ und „stimme eher zu“ wurden hier zusammengefasst.

anderen habe die vergleichsweise hohe Frauenerwerbsquote der DDR eine stärkere Gleichstellungsorientierung der Ostdeutschen bewirkt (vgl. etwa Braun et al.1994; Endrikat 2003, S. 127). Außerdem ist zu vermuten, dass die fortgeschrittene Säkularisierung 
zur Liberalisierung der Geschlechtsrollenvorstellung der ostdeutschen Bevölkerung beigetragen hat (s.u.).

\subsection{Theoretische Modelle zur Erklärung sexistischer Einstellungen}

Zur Erklärung individueller Geschlechtsrollenorientierungen werden in der Literatur für gewöhnlich zwei theoretische Konzepte unterschieden: Das interest-based-Modell und das exposure-based-Modell (vgl. Bolzendahl und Myers 2004; Lee et al. 2007; Davis und Greenstein 2009). Nach dem interest-based-Modell nehmen Individuen traditionelle (oder nicht-traditionelle) Geschlechtsrollenvorstellungen an, wenn sie selbst von traditionellen (oder von nicht-traditionellen) Rollenverteilungen profitieren. Dabei kann es sich z. B. um einen ökonomischen Gewinn, um einen Zuwachs an Prestige, Selbstbewusstsein oder Selbstverwirklichung etc. handeln. Berücksichtigt man etwa die jeweiligen Interessenstrukturen der Geschlechter, dann sollten Frauen im Vergleich zu Männern durchschnittlich häufiger egalitäre Rollenvorstellungen vertreten, da diese u. a. zur Beseitigung von Gehaltsdiskriminierungen beitragen (vgl. Bolzendahl und Myers 2004, S. 761). Das exposure-based Modell hingegen geht davon aus, dass die individuellen Geschlechtsrollenvorstellungen von den Einflüssen abhängen, denen Menschen in ihrer Sozialisation, der Ausbildung oder in anderen Kontexten ausgesetzt sind oder waren. Personen z. B., denen im Elternhaus eine sehr traditionelle Rollenverteilung vorgelebt wurde, sollten nach dem exposure-based-Modell seltener egalitäre Geschlechtsrollenvorstellungen entwickeln.

Beide Modelle erlauben Vorhersagen darüber, wie individuelle Merkmale sich auf die Geschlechtsrollenorientierungen auswirken. Allerdings besteht dabei das Problem, dass sich nicht alle relevanten Merkmale exklusiv einem der beiden Modelle zuordnen lassen und damit nicht immer trennscharf sind (vgl. Bolzendahl und Myers 2004, S. 763). So kann beispielsweise höhere Schulbildung einen negativen Einfluss auf sexistische Einstellungen ausüben, weil Menschen, die sich in höheren Bildungsinstitutionen bewegen eher einer egalitären Atmosphäre ausgesetzt sind (exposure-based-Modell). Im Hinblick auf Frauen lässt sich in diesem Kontext aber ebenso interessenbasiert argumentieren, weil mit zunehmender Bildung die Berufschancen von Frauen steigen (interest-based-Modell).

\subsection{Bestimmungsgründe und empirische Befunde zur Erklärung sexistischer} Einstellungen

Aus der Perspektive des exposure-based-Modells sind für die individuellen Rollenorientierungen zunächst einmal die Sozialisationseinflüsse in Kindheit und Jugend von Bedeutung. Als wichtige Faktoren für die Ausbildung egalitärer Geschlechtsrolleneinstellungen werden ein hohes Bildungsniveau der Eltern (insbesondere der Mutter) sowie die Berufstätigkeit der Mutter genannt. Kommen Heranwachsende durch höhere Bildung der Eltern früh mit egalitären Geschlechtsrollenvorstellungen in Kontakt und erleben ihre Mütter als erwerbstätig, so fällt es ihnen generell leichter, eine Berufstätigkeit von Frauen oder egalitäre Geschlechtsrollen als selbstverständlich wahrzunehmen (vgl. Tomeh 1979; Banaszak und Plutzer 1993; Rhodebeck 1996). 
Neben den Sozialisationseinflüssen im Elternhaus spielt bei der Genese der Geschlechtsrollenvorstellungen vor allem die eigene Schulbildung eine große Rolle. Mit dem exposure-based-Modell lässt sich argumentieren, dass Bildung und Religion in den meisten Gesellschaften zu den wichtigsten sozialen Institutionen gehören, denen Individuen ausgesetzt sind. In ihrer Wirkung auf das Geschlechterverhältnis unterscheiden sich allerdings Religion und Bildungssystem. Während die (säkularen) Bildungseinrichtungen Geschlechterungleichheiten zumeist infrage stellen und die Gleichstellung von Männern und Frauen tendenziell fördern (vgl. Glick et al. 2002), werden in den traditionellen Religionen Geschlechterungleichheiten oft im Namen Gottes legitimiert. So wird in der Geschichte des Christentums das Verhältnis der Geschlechter immer wieder als hierarchisch beschrieben und Frauen in etlichen Passagen des Schrifttums als minderwertig oder untergeordnet dargestellt. ${ }^{7}$ Findet im Zuge der Sozialisation eine Transmission dieser Weltsicht statt, so sollten sich im Hinblick auf das Geschlechterverhältnis stärker traditionelle Vorstellungen entwickeln. Empirische Ergebnisse, wonach stark religiöse Menschen eher konventionelle Geschlechtsrollenauffassungen vertreten (z. B. Thornton et al. 1983; Glick et al. 2002), unterstützen diese theoretischen Überlegungen. Dementsprechend traditionell sind auch die Geschlechterverhältnisse in stark religiös geprägten Gesellschaften und zwar nicht nur in christlichen, sondern auch in islamischen Gesellschaften oder im orthodoxen Judentum.

Bildung hingegen, so zeigt die empirische Vorurteilsforschung in vielen Fällen, geht mit einer Abnahme von Vorurteilen im Allgemeinen (vgl. Hopf 1999; Rippl 2002; Heyder 2003) und sexistischen Einstellungen im Besonderen einher (Thornton et al. 1983, Davis und Robinson 1991; Rhodebeck 1996; Bryant 2003; Bolzendahl und Myers 2004). ${ }^{8}$ Der negative Zusammenhang zwischen Bildung und traditionellen Geschlechtsrollenvorstellungen lässt sich über verschiedene Mechanismen erklären, die zum großen Teil dem exposure-based-Modell zugeordnet werden können. Zum einen nehmen kognitive Fähigkeiten und Wissensbestände mit steigender Bildung zu. Sie versetzen den Menschen in die Lage, gesellschaftliche Strukturen leichter zu durchschauen (Hopf 1999) und stereotype Geschlechtsrollen kritisch zu hinterfragen. Zum anderen gilt Schule als wichtige Sozialisationsinstanz für die Ausbildung von Wertorientierungen (Hopf 1999; Hadjar 2006; Mays 2009). So konnte etwa gezeigt werden, dass höhere Bildungseinrichtungen eine zentrale Rolle bei der Vermittlung demokratischer Werte einnehmen (Hopf 1999; Hadjar 2006). Zurückgeführt werden diese Effekte im Wesentlichen auf eine andere, meist demokratischere Lernkultur der oberen Bildungswege sowie auf weiterführende

7 Auch heute noch fällt es den christlichen Kirchen schwer, Frauen als gleichberechtigt anzuerkennen (wie z. B. das Weiheverbot für Frauen in der katholischen Kirche zeigt). Die evangelischen Kirchen in Deutschland präsentieren sich heute zwar deutlich progressiver, sieht man von den evangelikalen Gruppen ab, die formale Gleichstellung von Theologen und Theologinnen aber wurde auch hier erst Ende des letzten Jahrhunderts vollzogen. In den USA zeigen empirische Befunde zudem, dass konservative Protestanten von allen untersuchten Religionsgruppen die stärksten traditionellen Geschlechtsrollenvorstellungen aufweisen (vgl. Bolzendahl und Myers 2004).

8 Allerdings wurde in diesem Zusammenhang die Hypothese formuliert, dass formal hoch Gebildete nicht unbedingt weniger Vorurteile als Personen mit weniger Schulbildung hätten, sondern nur besser in der Lage seien, ihre Vorurteile zu vertuschen (vgl. Hopf 1999). 
Lerninhalte oder Auseinandersetzung mit neuen Ideen und längere Verweildauer in den entsprechenden Bildungseinrichtungen (vgl. z. B. Hopf 1999; Hadjar 2006).

Für Frauen lässt sich, im Sinne des interest-based-Modells, ein weiteres Argument für den Zusammenhang zwischen Bildung und Geschlechtsrollen anführen. Frauen entwickeln mit steigender Bildung weiterführende Berufsaspirationen und Kompetenzen und haben damit zusätzlich ein starkes eigenes Interesse an egalitären Geschlechtsrollen.

Dementsprechend gilt in der Literatur der Zusammenhang zwischen weiblicher Berufstätigkeit und egalitären Einstellungen als ein robuster Befund (vgl. z. B. Thornton et al. 1983; Bolzendahl und Myers 2004). Diese Beziehung kann zum einen dadurch erklärt werden, dass erwerbstätige Frauen ökonomisch unabhängiger sind und somit stark von einer Berufstätigkeit profitieren (interest-based-Modell). Zum anderen ermöglicht die weibliche Partizipation am Arbeitsmarkt ein Aushandeln der familiären Arbeitsteilung, was tendenziell zum Aufbrechen traditioneller Rollenaufteilungen führen kann. Darüber hinaus sind berufstätige Frauen eher egalitären Vorstellungen ausgesetzt und entwickeln durch die Erfahrung im Beruf oft ein stärkeres Bewusstsein für Diskriminierungen (exposure-based-Modell, vgl. zusammenfassend Bolzendahl und Myers 2004). In diesem Zusammenhang ist allerdings zu berücksichtigen, dass die Kausalitätsrichtung der Beziehung zwischen weiblicher Berufstätigkeit und Geschlechtsrollenorientierungen nicht eindeutig geklärt ist (vgl. Lee et al. 2007). So lässt sich argumentieren, dass Frauen seltener einer Berufstätigkeit nachgehen, wenn sie über traditionelle Rollenvorstellungen verfügen. Ein ähnliches Problem stellt sich im Kontext der Familienstruktur. So ist bekannt, dass verheiratete und kinderreiche Personen über traditionellere Vorstellungen verfügen als jene, die unverheiratet (oder geschieden) und kinderlos sind (Plutzer 1991). Hier ließe sich analog vermuten, dass Frauen, die über traditionelle Rollenvorstellungen verfügen auch eher heiraten und Kinder bekommen und seltener einer Berufstätigkeit nachgehen.

Eine wichtige Determinante für die Erklärung sexistischer Einstellungen ist das Lebensalter: Ältere Personen verfügen durchweg über traditionellere Vorstellungen als junge Menschen (Davis und Robinson 1991; Brewster und Padavic 2000). Interpretiert wird dieser Befund häufig als Kohorten- oder Sozialisationseffekt infolge der gesellschaftlichen Modernisierungsbewegung. Ältere Menschen, die ihre formative Phase in einer Gesellschaft traditioneller Rollenverteilung erfahren haben, entwickelten dementsprechend dauerhaft stärker antiegalitäre Geschlechtsrollen als jüngere, die unter weniger traditionellen Bedingungen aufwuchsen (exposure-based-Modell). Für Frauen spielt in diesem Kontext auch das Interessen- Argument eine Rolle. So profitieren jüngere Frauen stärker von egalitären Geschlechtsrollenvorstellungen als ältere Frauen, da sie über eine bessere Bildung verfügen, häufiger berufstätig sind usw.

Neben den hier aufgeführten Determinanten sollten bei der Erklärung traditioneller Geschlechtsrollenvorstellungen die Aspekte Wertorientierungen, Wahrnehmung ökonomischer Deprivation sowie Anomia berücksichtigt werden, die bei der Erforschung von Geschlechtsrollenvorstellungen bislang keine oder nur wenig Beachtung gefunden haben.

Aus theoretischer Sicht dürften übergeordnete, grundlegende Werte hinsichtlich der sozialen Ordnung einer Gesellschaft eine besondere Bedeutung für die Entwicklung der Geschlechtsrollenauffassung haben. Werden grundsätzlich im Sinne einer allgemeinen und übergeordneten Werthaltung egalitäre Werte unterstützt und soziale Hierarchien oder Ungleichheit abgelehnt, dann ist davon auszugehen, dass zur Vermeidung kogni- 
tiver Dissonanzen (Festinger 1957) auch in speziellen Einstellungsbereichen wie den Geschlechtsrollenvorstellungen egalitäre Haltungen präferiert werden (vgl. Davis und Greenstein 2009). Werden hingegen soziale Gruppen prinzipiell hierarchisch geordnet oder als nicht gleichwertig betrachtet und die daraus resultierenden Ungleichheiten als legitim oder gar als wünschenswert angesehen, dann sind umgekehrt auch antiegalitäre Geschlechtsrolleneinstellungen wahrscheinlich (vgl. das verwandte Konzept der Sozialen Dominanzorientierung nach Sidanius und Pratto 1999).

Zum Zusammenhang zwischen der Unterstützung egalitärer Werte und Schulbildung liegen allerdings konkurrierende Hypothesen vor (vgl. Hadjar 2008). Einerseits wird behauptet, dass mit zunehmender Bildung die Akzeptanz sozialer Ungleichheiten steigt, da besser Gebildete in der Regel privilegiertere Positionen erreichen und deshalb bestrebt sind, diese zu erhalten oder zu legitimieren. Andererseits wird argumentiert, höher Gebildete seien dank ausgeprägter kognitiver Fähigkeiten und durch den Kontakt mit neuen Ideen eher imstande, soziale Ungleichheit kritisch zu reflektieren (s. Abschnitt zur Wirkung von Bildung auf Geschlechtsrollenorientierung).

Übertragen auf Geschlechtsrollen könnte man hier dem interest-based-Modell gemäß vermuten, dass insbesondere hochgebildete und ökonomisch privilegierte Männer ein Interesse an klassischen Rollenverteilungen haben. Denn bei einer traditionellen Arbeitsaufteilung, bei der die Partnerin den häuslichen Arbeitsbereich übernimmt, können sich diese Männer stärker auf ihre berufliche Karriere konzentrieren, auf ein zweites Einkommen sollten solche Haushalte der Regel nicht angewiesen sein. Zudem müssen die betreffenden Männer bei einer derartigen Rollenverteilung weniger Konkurrenz von Frauen auf dem Arbeitsmarkt fürchten. Diesem Interessenargument kann allerdings aus Sicht des exposure-based-Modells entgegengehalten werden, dass hochgebildete oder privilegierte Männer in ihrer Sozialisation oder Ausbildung eher liberalen Einflüssen ausgesetzt waren. Darüber hinaus haben es diese Männer vermutlich häufiger mit Frauen zu tun, die egalitäre Geschlechtsrollenvorstellungen vertreten. Nach Abwägung der verschiedenen Argumente dürften letztere schwerer wiegen, sodass damit zu rechnen ist, dass hochgebildete oder privilegierte Männer eher liberale Geschlechtsrollenvorstellungen favorisieren.

Die Wahrnehmung wirtschaftlicher Benachteiligung schließlich kann, ebenso wie gesellschaftliche Desintegration angesichts einer als unsicher und bedrohlich empfundenen Welt, zur Verunsicherung und zu einem verstärkten Sicherheits- und Ordnungsbedürfnis führen. Eine klare gesellschaftliche Rollenzuteilung nach traditionellem Muster könnte dann zur Reduktion der Unsicherheits- und Bedrohungsgefühle beitragen.

\subsection{Hypothesen}

Aus den skizzierten theoretischen Überlegungen und empirischen Befunden lassen sich nun mehrere, mit dem ALLBUS 2008 überprüfbare Hypothesen ableiten.

Bei der Erklärung sexistischer Einstellungen ist dabei sowohl von direkten als auch indirekten Einflussfaktoren auszugehen. Von indirekten Effekten wird gesprochen, wenn Einflussgrößen nicht direkt, sondern vermittelt über weitere Variablen wirksam werden. Solche Effekte sind vor allem bei den zeitlich lang zurückliegenden Faktoren der Sozialisation zu erwarten. Die familiale Sozialisation etwa oder der soziokulturelle Einfluss unterschiedlicher Epochen und Gesellschaftssysteme sollten größtenteils indirekt, ver- 
mittelt über die Ausbildung genereller Wert-, Einstellungs- und Kompetenzstrukturen, wirksam werden. Direkte Effekte sollten insbesondere von Bedingungen der Situation ausgehen. Angesprochen sind hier die gegenwärtigen Lebensbedingungen oder Wahrnehmungen der Betroffenen.

Die oben dargestellten Ost-West-Unterschiede in den Geschlechtsrollenauffassungen lassen einen insgesamt deutlichen Effekt der Sozialisation vermuten. ${ }^{9}$ Neben der Sozialisationswirkung der jeweiligen politischen Systeme oder gesellschaftlichen Epochen sollte auch die Sozialisation im Elternhaus eine Rolle spielen. Sowohl die religiöse Sozialisation als auch das elterliche Bildungsniveau dürften einen mittelbaren Einfluss auf die Geschlechtsrollenorientierung der Kinder ausüben. Konkret ist zu erwarten, dass Personen, die ihre primäre Sozialisation in den ostdeutschen Landesteilen erlebten, seltener sexistische Einstellungen aufweisen als solche, die ihre Jugend in westdeutschen Ländern verbracht haben (Hypothese H1). Wer in der Zeit vor Beginn der gesellschaftlichen Modernisierung und Frauenbewegung aufgewachsen ist, sollte dagegen über deutlich traditionellere Geschlechtsrollenvorstellungen verfügen (Hypothese H2). ${ }^{10}$ Ebenfalls sichtbare Effekte sollten von der Sozialisation im Elternhaus ausgehen: Je religiöser die Eltern in der Jugend waren (Hypothese H3) oder je niedriger das elterliche Bildungsniveau (Hypothese H4) war, desto traditioneller sollten die Geschlechtsrollenorientierungen der Nachfahren im Erwachsenenalter sein. Darüber hinaus ist zu vermuten, dass eine mütterliche Berufstätigkeit während der Jugendzeit Spuren bei den Geschlechtsrollenvorstellungen der Nachkommen hinterlässt. Es ist damit zu rechnen, dass Personen seltener sexistische Einstellungen ausbilden, wenn die eigene Mutter berufstätig war (Hypothese H5) und eine Berufstätigkeit von Frauen somit leichter als selbstverständlich wahrgenommen werden konnte.

Neben diesen direkten und indirekten Sozialisationswirkungen ist von Determinanten auszugehen, die sich vor allem direkt und kurzfristig auf Geschlechtsrollenorientierungen auswirken. Bildung (Hypothese Ho) sollte aus den oben ausgeführten Überlegungen heraus den bekannten negativen Einfluss auf traditionell sexistische Orientierungen ausüben. Eine Berufstätigkeit von Frauen (Hypothese $\mathrm{H7a}$ ) oder der Partnerinnen der männlichen Befragten (Hypothese $H 7 b$ ) sollte egalisierende Wirkung zeigen und ebenfalls zur Reduktion sexistischer Einstellungen beitragen. Diese These geht davon aus, dass eine Berufstätigkeit von Frauen (oder Partnerinnen) sozialisierend wirkt und einen negativen Einfluss auf die traditionelle Geschlechtsrollenorientierung hat. Die umgekehrte Kausalrichtung ist allerdings ebenfalls plausibel (s. o.). Die Berufstätigkeit von Frauen oder Partnerinnen wird dennoch in das Modell mit einbezogen, um die Stärke der Beziehung zum Sexismus in Relation zu den anderen Variablen einschätzen zu können. ${ }^{11}$ Religiosi-

9 Allerdings müssen die beobachteten Ost-West-Unterschiede nicht zwingend und nicht ausschließlich auf Sozialisationseffekte zurückgehen. Bestehende Ungleichheiten in den Lebensbedingungen können ebenfalls Ursache der differierenden Rollenvorstellungen sein (s. dazu auch weiter unten).

10 Es wird davon ausgegangen, dass nicht nur in der BRD sondern auch in der DDR seit Mitte der 1960er Jahre ein Prozess der gesellschaftlichen Modernisierung stattgefunden hat.

11 Das Endogenitätsproblem ist in Strukturgleichungsmodellen nicht so relevant, wenn möglich ist, Messfehlerkorrelationen als zusätzliche Parameter zuzulassen. In den vorliegenden Model- 
Tab. 1: Hypothesen im Überblick

\begin{tabular}{lll}
\hline Hypothese & Konstrukt & Vermutete Wirkung \\
\hline H1 & Sozialisation in Ostdeutschland & Reduziert traditionellen Sexismus \\
H2 & Kohorte vor 1949 geboren & Fördert traditionellen Sexismus \\
H4 & Religiosität Eltern & Fördert traditionellen Sexismus \\
H5 & Bildung Eltern & Reduziert traditionellen Sexismus \\
H6 & Berufstätigkeit Mutter & Reduziert traditionellen Sexismus \\
H7a & Eigene Bildung & Reduziert traditionellen Sexismus \\
H7b & Eigene Berufstätigkeit (bei Frauen) & Reduziert traditionellen Sexismus \\
& Berufstätigkeit Partnerin (bei & Reduziert traditionellen Sexismus \\
H8 & Männern) & \\
H9 & Eigene Religiosität & Fördert traditionellen Sexismus \\
H10 & Antiegalitäre Haltung & Fördert traditionellen Sexismus \\
& Wahrnehmung ökonomischer & Fördert traditionellen Sexismus \\
H11 & Deprivation & \\
& Anomia & Fördert traditionellen Sexismus
\end{tabular}

tät (Hypothese $H 8$ ) und antiegalitäre Werthaltungen (Hypothese $H 9$ ) sowie die Wahrnehmung ökonomischer Deprivation (Hypothese H10) und Anomia (Hypothese H11) schließlich sollten traditionelle Rollenbilder verstärken.

Tabelle 1 zeigt die Hypothesen noch einmal im Überblick:

Der anschließende Hypothesentest wird für Männer und Frauen getrennt durchgeführt, da mit den Hypothesen $7 a$ und $7 b$ jeweils unterschiedliche Variablen (eigene Erwerbstätigkeit bei Frauen oder Erwerbstätigkeit der Partnerin bei Männern) geprüft werden. Die nach Geschlechtern getrennte Modellberechnung ermöglicht zudem eine eventuelle Aufdeckung geschlechtsspezifischer Unterschiede in der Kausalstruktur zur Erklärung des Sexismus.

Die folgenden Pfaddiagramme (Abb. 4 für Männer, Abb. 5 für Frauen) stellen die vermuteten Beziehungen zwischen den theoretischen Konstrukten grafisch dar. Sie geben die Kausalstrukturen entsprechend der statistischen Modellierung des Ausgangsmodells wieder. Die Doppelpfeile stehen für Korrelationen zwischen Faktoren, die einfachen Pfeile stehen für gerichtete Effekte.

\section{Analysedesign und Datengrundlage}

Die Überprüfung der postulierten Zusammenhänge erfolgt unter Verwendung zweier Strukturgleichungsmodelle auf Basis der ALLBUS-Daten aus dem Jahr 2008. Für die vorliegende Fragestellung bieten Strukturgleichungsmodelle einige Vorteile: Zum einen können mit diesem Verfahren mehrere abhängige Variablen simultan formuliert werden, die gleichzeitig als erklärende Einflussgrößen für andere Variablen fungieren. Das bedeutet, dass neben direkten Einflüssen auch indirekte Effekte auf die Zielvariablen ermittelt

len wird allerdings die Berufstätigkeit von Frauen über nur einen Indikator erfasst, was zu technischen Problemen bei der Berechnung der Modelle führt. 


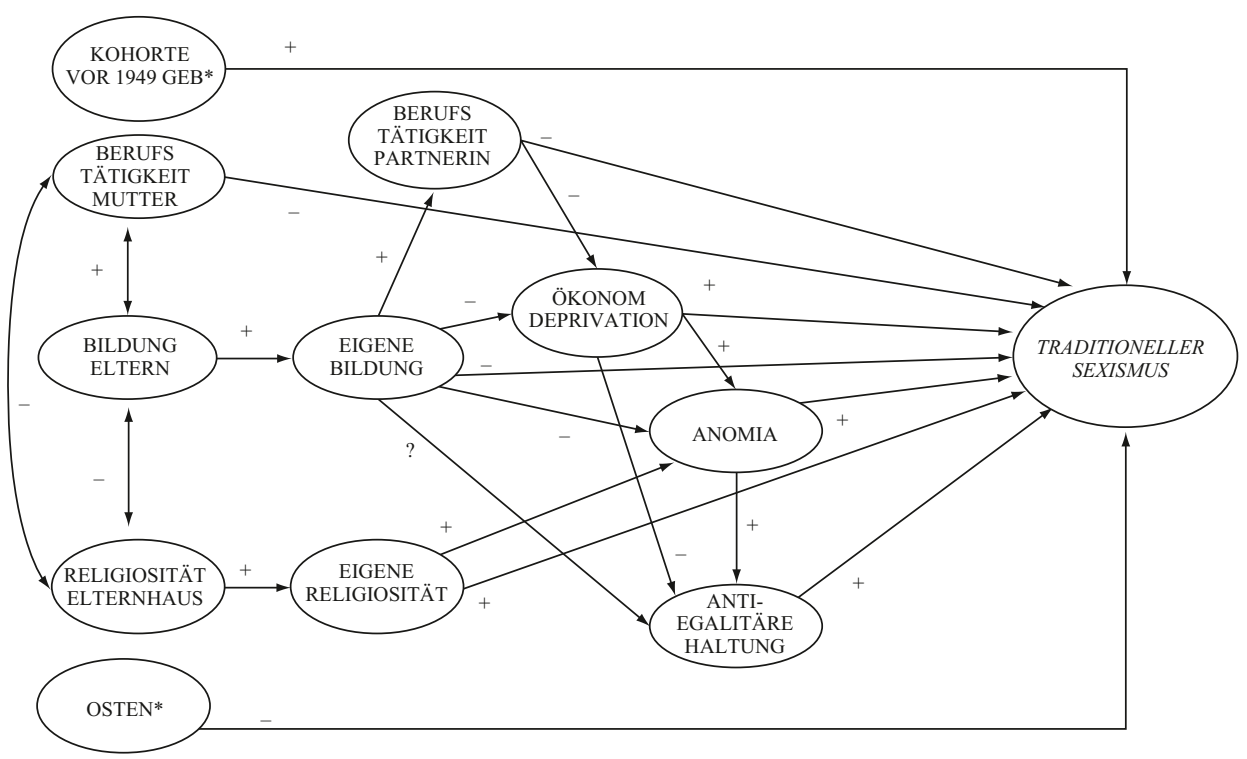

*Weitere vermutete Effekte bzw. Kovarianzen zwischen den Variablen „Osten“ bzw. „Kohorte vor 1949 geboren“ und den restlichen erklärenden Faktoren werden der Übersichtlichkeit wegen in der Grafik nicht dargestellt.

Abb. 4: Theoretisches Ausgangsmodell für Männer

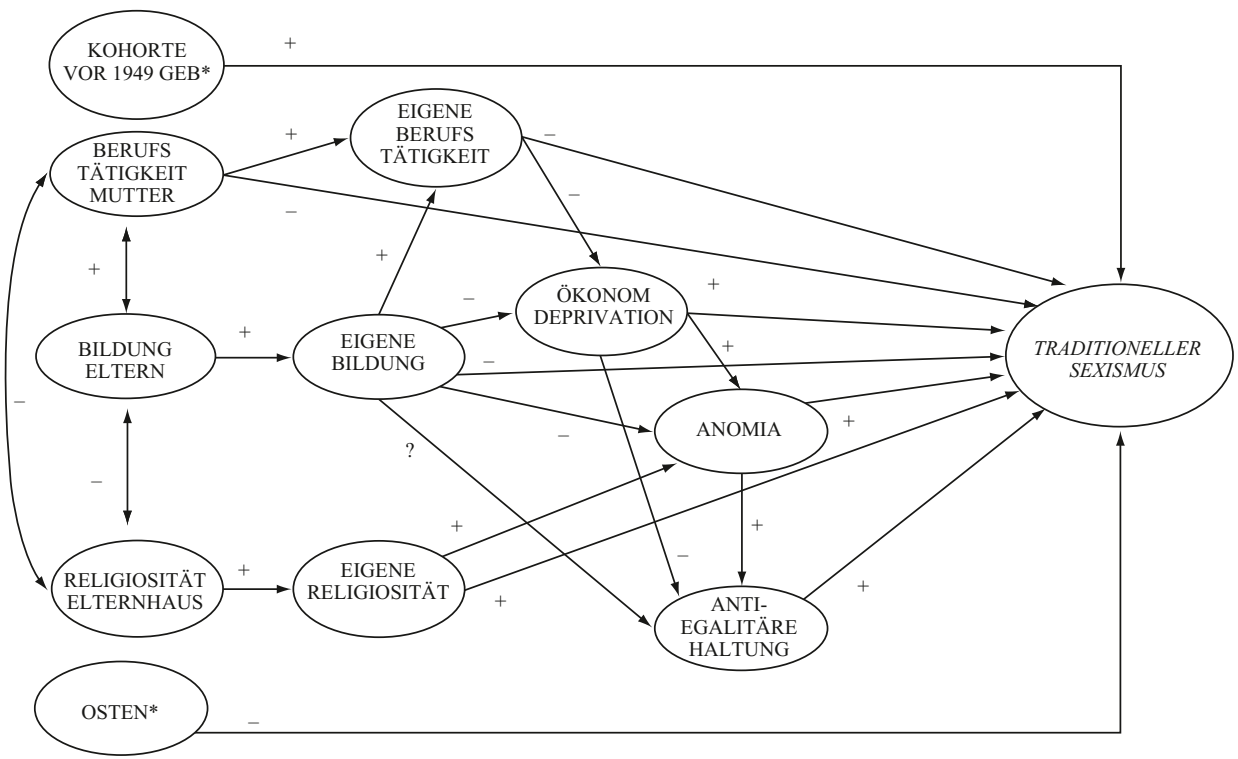

*Weitere vermutete Effekte bzw. Kovarianzen zwischen den Variablen „Osten“ bzw. „Kohorte vor 1949 geboren“ und den restlichen erklärenden Faktoren werden der Übersichtlichkeit wegen in der Grafik nicht dargestellt.

Abb. 5: Theoretisches Ausgangsmodell für Frauen 
werden können. Durch Addition der direkten und indirekten Effekte lässt sich zusätzlich der sogenannte totale Effekt einer erklärenden auf eine abhängige Zielgröße ermitteln. Zum anderen verwenden Strukturgleichungsmodelle mit latenten Variablen multiple Indikatoren zur Erfassung der theoretischen Konstrukte (vgl. Bollen 1989; Reinecke 2005). Mit Hilfe eines solchen multiplen Indikatorenmodells wird versucht, die Fehlerhaftigkeit einer Messung zu reduzieren, indem verschiedene Indikatoren zur gemeinsamen Messung einer latenten Variablen herangezogen werden. Sofern möglich, sollten also die latenten Variablen über mehrere Indikatoren erfasst werden. In der vorliegenden Analyse mit den Daten des ALLBUS 2008 ist dies für einige, nicht aber für alle latenten Variablen möglich. In diesen Fällen muss auf die Berücksichtigung der Messfehler verzichtet werden. ${ }^{12}$

\subsection{Operationalisierung}

Zur Messung des abhängigen Konstrukts traditioneller Sexismus werden die drei klassischen Items „Frau soll lieber Mann bei der Karriere helfen“, „Frau soll zu Hause bleiben und Kinder versorgen“ und „Frau soll nach Heirat Arbeitsplatz freimachen“ verwendet. ${ }^{13}$

Als erklärende Sozialisationsvariable, die den sozio-kulturellen Sozialisationseinfluss der ostdeutschen Landesteile messen soll, wird die Frage nach den Bundesländern, in denen die Befragten während ihrer Jugendzeit vorwiegend gelebt haben, herangezogen. Um zu überprüfen, ob ein Kohorteneffekt, d.h. ein Effekt der „formativen Phase“ vorliegt, wird eine dichotome Kohortenvariable kodiert, die Auskunft darüber gibt, ob die Befragten vor 1949 oder danach geboren wurden. Die Vorstellung einer „formativen Phase“" geht davon aus, dass in der Zeit der mittleren Adoleszenz (d.h. im Alter von etwa 15/16 Jahren) wesentliche Lernprozesse stattfinden, die entscheidend für die Entwicklung gesellschaftlicher Orientierungen sind. Die dichotome Altersvariable gibt also darüber Auskunft, ob die Personen ihre „formative“ Phase ab etwa Mitte der 1960er Jahre hatten oder vorher, d.h. in der Zeit vor der gesellschaftlichen Modernisierung. Drei weitere Konstrukte betreffen Sozialisationseinflüsse des Elternhauses. Die erklärende latente Variable Religiosität der Eltern wird über die Kirchgangshäufigkeit beider Elternteile erfasst. Die allgemeine Schulbildung beider Eltern bildet das Konstrukt Bildung der Eltern. Die Frage, ob die Mutter während der Jugend der Befragten berufstätig war, wird schließlich über die Variable „Erwerbstätigkeit“" gemessen.

Die Operationalisierung der Konstrukte eigene Religiosität und eigene Bildung entspricht derjenigen der Eltern. Religiosität wird über die Kirchgangshäufigkeit (ein Indi-

12 Die Schätzung des Strukturgleichungsmodells erfolgt mithilfe der Full-Information ML-Methode (FIML), die im Falle von Missing Completelyatrandom (MCAR) oder Missingatrandom (MAR) eine Parameterschätzung mit den üblichen Gütekriterien der Maximum-LikelihoodSchätzer (asymptotische Erwartungstreue, Konsistenz, asymptotische Effizienz - vgl. Bollen 1989, S. 108) erlaubt und anderen Methoden zum Umgang mit fehlenden Werten überlegen ist (Arbuckle 1996; Reinecke 2005). Für die folgende Analyse wird angenommen, dass die Missing at random - Bedingung vorliegt, d. h. das Fehlen von Werten ist von den beobachteten Daten abhängig.

13 Eine Übersicht über die Itemformulierungen und Kodierungen befindet sich im Anhang in der Tab. 3 . 
kator) und Bildung über die allgemeine Schulbildung (ebenfalls ein Indikator) bestimmt. Auch die Frage, ob die weiblichen Befragten (oder die Partnerinnen der Befragten) einer Berufstätigkeit nachgehen, wird über die Variable „Erwerbstätigkeit" ${ }^{\text {"14 }}$ gemessen.

Das theoretische Konstrukt wahrgenommene ökonomische Deprivation wird über die Items „Bewertung der eigenen wirtschaftlichen Lage“ und „,erhalte gerechten Anteil am Lebensstandard“ operationalisiert. Für die Operationalisierung der beiden letzten latenten Variablen stehen ebenfalls jeweils zwei Items zur Verfügung: Zur Messung von Anomia werden die Items „Politiker uninteressiert an einfachen Leuten“ und „,bei dieser Zukunft keine Kinder mehr“ herangezogen. Das Konstrukt Antiegalitarismus schließlich wird über die Variablen „,soziale Unterschiede sind gerecht“ und „Rangunterschiede sind akzeptabel“" abgebildet.

\section{Ergebnisse}

In den Abb. 6 und 7 sind jeweils die Koeffizienten der geschätzten, angepassten Strukturgleichungsmodelle abgebildet. Die Doppelpfeile geben die Korrelationskoeffizienten zwischen den betreffenden Konstrukten wieder. Bei den gerichteten Pfeilen handelt es sich um die gerichteten standardisierten Effekte. Die Modellanpassung ist in beiden Fällen zufriedenstellend: Im Modell für Männer beträgt der Chiquadratwert 218,85 bei 114 Freiheitsgraden $(\mathrm{p}=0,00)$, der RMSEA liegt bei 0,023 ( $\mathrm{p}-\mathrm{Close}=1,00)$.

Für das Frauen-Modell wird ein Chiquadratwert von 194,26 bei 117 Freiheitsgraden $(p=0,00)$ erreicht. Der der RMSEA liegt hier bei 0,019 ( $p-C l o s e=1,00)$. Auch die Erklärungsleistung der Modelle insgesamt ist mit einem $\mathrm{R}^{2}$ von $39 \%$ für Männer und von $45 \%$ für Frauen recht gut. ${ }^{15}$

Das angepasste Strukturmodell für Männer (Abb. 6) zeigt, dass die geschätzten direkten Effekte im Hinblick auf die Zielvariable Sexismus in allen Fällen den theoretischen Erwartungen entsprechen. Die Zugehörigkeit zur Kohorte der vor 1949 Geborenen, (elterliche und eigene) Religiosität, antiegalitäre Haltungen, die Wahrnehmung von ökonomischer Deprivation sowie Gefühle von Anomia verstärken traditionelle Geschlechtsrollenorientierungen bei Männern. Die Prädiktoren (elterliche und eigene) Bildung, Sozialisation in Ostdeutschland sowie Berufstätigkeit der Mutter in der Jugend der Befragten oder Erwerbstätigkeit der Partnerin ${ }^{16}$ hingegen reduzieren erwartungsgemäß sexistische Einstellungen.

14 Die Variablen „eigene Erwerbstätigkeit“ oder „Erwerbstätigkeit Partnerin“ wurden als dichotome Variablen kodiert, die den Wert 1 erhalten, wenn eine Erwerbstätigkeit vorliegt und den Wert 0, wenn keine Erwerbstätigkeit ausgeübt wird. Befragte ohne feste Partnerin bekamen hier den Wert 0 zugewiesen.

15 Um eine bessere Modellanpassung zu erreichen, wurden für beide Modelle jeweils vier Nebenladungen freigegeben. Die Beta-Koeffizienten blieben trotz dieser Modifikation stabil. Der Übersichtlichkeit wegen sind in den Grafiken keine Indikatorenladungen der Messmodelle aufgeführt. Die einzelnen standardisierten Indikatorenladungen sowie die Residualvarianzen können Tab. 4 im Anhang entnommen werden.

16 Die endogene Variable „Berufstätigkeit der Partnerin“ oder „Eigene Berufstätigkeit“ (bei Frauen) haben nur zwei Ausprägungen. Dies ist in den Schätzmodellen mit gewissen Proble- 


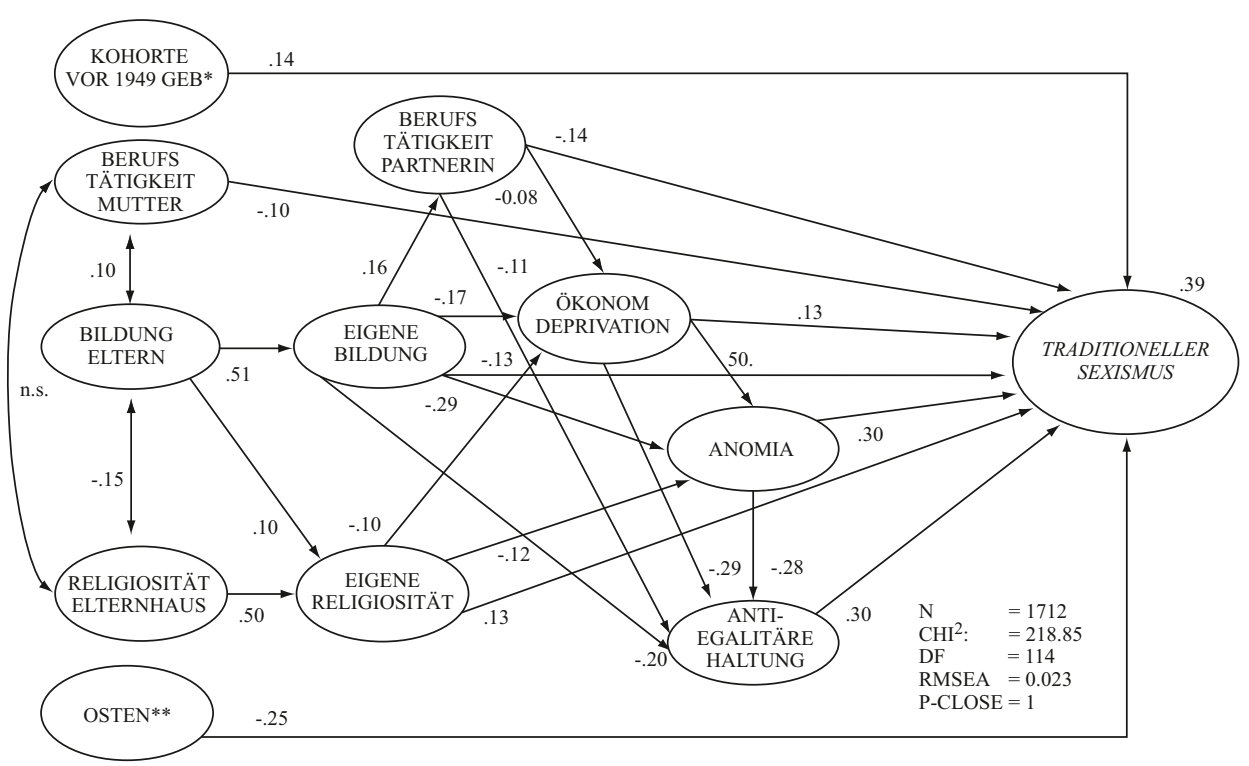

*Von der Variable „Kohorte vor 1949 geboren“ gehen auf die übrigen erklärenden Konstrukte folgende standardisierten und signifikanten Effekte aus: Religion Eltern=0,23, Bildung Eltern=-0,26, Beruf Mutter=-0,21, Ökonomische Deprivation=-0,19, Berufstätigkeit Partnerin=-0,31.

**Von der Variable „Osten“ gehen auf die übrigen erklärenden Konstrukte folgende standardisierten und signifikanten Effekte aus: Religion Eltern $=-0,53$, Bildung Eltern $=0,07$, Beruf Mutter $=0,35$, Eigene Religiosität $=-0,08$, Ökonomische Deprivation $=0,40$, Anomia $=0,10$, Anti-Egalitäre Haltung $=-0,13$, Berufstätigkeit Partnerin $=0,11$.

Abb. 6: Angepasstes Strukturmodell für Männer, standardisierte Koeffizenten

Für Frauen stellt sich die Situation etwas anders dar. So können zwei der formulierten Hypothesen nicht bestätigt werden (Abb. 7). Im Gegensatz zu Männern verringern sich sexistische Einstellungen bei Frauen nicht, wenn die Mutter einer Erwerbstätigkeit nachgegangen ist (Hypothese H5). ${ }^{17}$ Ebenfalls ohne Bedeutung ist bei Frauen die Perzeption ökonomischer Deprivation (H10). ${ }^{18}$ Frauen, die sich als ökonomisch benachteiligt emp-

men verbunden, da hier ein lineares Wahrscheinlichkeitsmodell unterstellt wird. Problematisch ist dabei, dass nicht garantiert werden kann, dass die Vorhersagewerte der Variablen zwischen 0 und 1 liegen. Außerdem wird die Homoskedastizitätsannahme bei diesen dichotomen endogenen Variablen verletzt. Gleichwohl können die Schätzungen als Annäherung an die tatsächlichen Beziehungen angenommen werden.

17 Worauf der hier beobachtete Unterschied zwischen den Geschlechtern zurückzuführen ist, lässt sich mit dem vorliegenden Datensatz nicht weiter untersuchen. Eine mögliche Erklärung wäre, dass Jungen vor allem dann zu Hausarbeiten herangezogen werden, wenn ihre Mütter berufstätig sind, während Mädchen unabhängig von einer mütterlichen Berufstätigkeit im Haushalt zu helfen haben. Lernen Jungen dann früh, dass Hausarbeit nicht per se „Frauensache“ und Berufstätigkeit nicht gleich „Männersache“ ist, so ist es plausibel, dass sie auch seltener sexistische Einstellungen entwickeln. Ob diese Hypothese zutrifft, müsste allerdings mit anderen Daten überprüft werden.

18 Wie in Tab. 2 zu sehen ist, zeigt sich für Frauen nur ein indirekter Effekt der wahrgenommenen ökonomischen Deprivation von 0,05. auf den Sexismus. Da dieser Wert unter der Marke von 0,10 liegt wird er als nicht substanziell angesehen. 


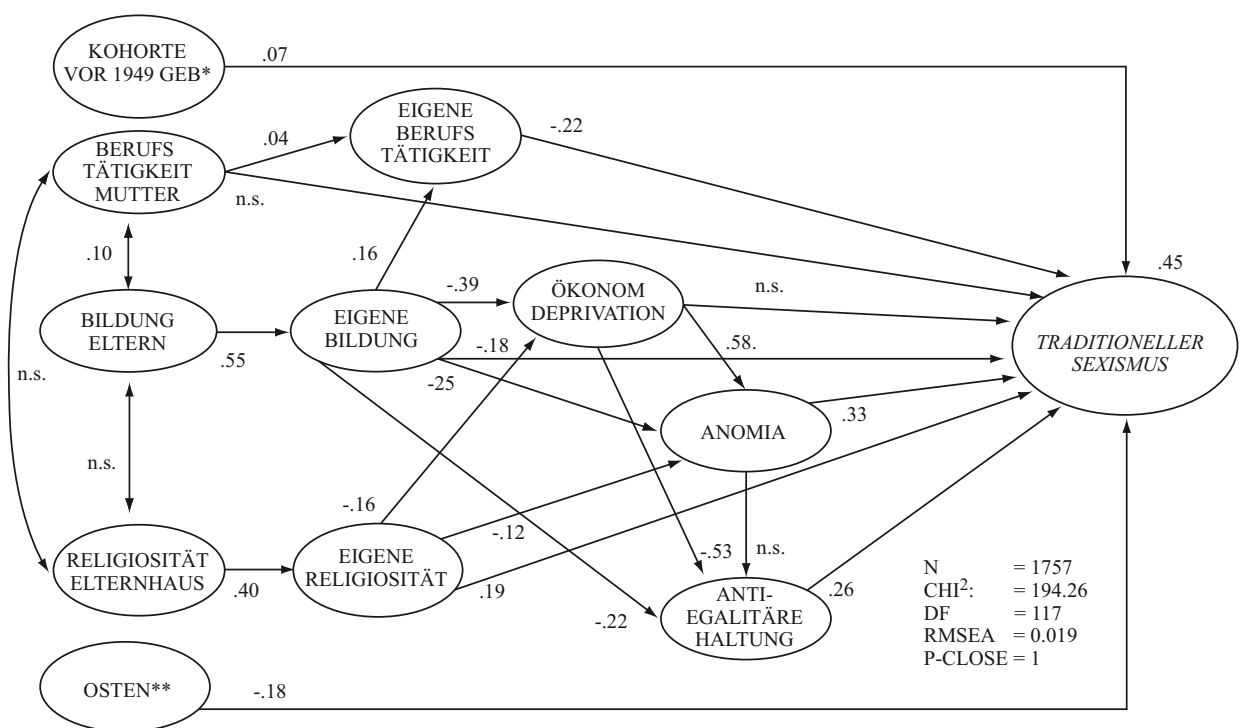

*Von der Variable „Kohorte vor 1949 geboren“ gehen auf die übrigen erklärenden Konstrukte folgende standardisierten und signifikanten Effekte aus: Religion Eltern $=0,25$, Bildung Eltern $=-0,19$, Beruf Mutter $=-0,20$, Eigene Religiosität $=0,07$, Eigene Bildung $=-0,22$, Ökonomische Deprivation=-0,22, Eigene Berufstätigkeit $=-0,56$.

**Von der Variable „Osten“ gehen auf die übrigen erklärenden Konstrukte folgende standardisierten und signifikanten Effekte aus: Religion Eltern=-0,46, Beruf Mutter=0,33, Eigene Religiosität $=-0,17$, Eigene Bildung=0,05, Ökonomische Deprivation=0,11, Anomia=0,10, Anti-Egalitäre Haltung $=-0,25$ Eigene Berufstätigkeit $=0,10$.

Abb. 7: Angepasstes Strukturmodell für Frauen, standardisierte Koeffizenten

finden, orientieren sich, anders als Männer, nicht stärker an traditionellen Geschlechtsrollen. Die Tatsache, dass dieser Faktor nur bei Männern von Belang ist, könnte auf eine Konkurrenzsituation hindeuten: Fühlen sich Männer wirtschaftlich schlechter gestellt, so ist denkbar, dass sie Frauen als Rivalinnen, etwa um Arbeitsplätze, wahrnehmen und daher die traditionelle Hausfrauenrolle eher befürworten. Die übrigen Hypothesen hingegen lassen sich auch für Frauen bestätigen.

Addiert man die direkten und indirekte Effekte und vergleicht die relative Erklärungskraft der verschiedenen Konstrukte miteinander (totale Effekte, Tab. 2), so zeigt sich, dass für Männer der Ort der Sozialisation (Sozialisation in Ostdeutschland: totaler Effekt = $-0,32)$ die insgesamt wichtigste Einflussgröße darstellt, dicht gefolgt von den Faktoren Antiegalitarismus (totaler Effekt $=0,30$ ) und Bildung (totaler Effekt $=-0,28$ ). Das Alter in Form der Kohortenzugehörigkeit (totaler Effekt $=0,24$ ) und Effekte der Situation wie Anomia oder das Empfinden ökonomischer Deprivation (totale Effekte $=0,21$, und 0,15) spielen bei der Erklärung sexistischen Einstellungen von Männern dagegen eine etwas geringere Rolle.

Darüber hinaus scheint bei den sozial-strukturellen Determinanten auch die Berufstätigkeit der Partnerin eine Rolle zu spielen (totaler Effekt $=-0,18$ ) und bei Männern $\mathrm{zu}$ etwas liberaleren Geschlechtsrollenvorstellungen zu führen. ${ }^{19}$ Ebenfalls signifikant,

19 Dieser Effekt sollte allerdings nur unter Vorbehalt angenommen werden, da die Kausalrichtung der Variablen letztlich nur mithilfe von Längsschnitt-Daten geklärt werden kann. 
Tab. 2: Totale und in Klammern direkte und indirekte, standardisierte Effekte auf das Konstrukt Sexismus.* (Quelle: ALLBUS 2008, eigene Berechnungen.)

\begin{tabular}{lll}
\hline & Sexismus bei Männern & Sexismus bei Frauen \\
\hline $\begin{array}{l}\text { Hintergrundkonstrukte der } \\
\text { Sozialisation }\end{array}$ & & \\
Sozialisation in Ostdeutschland & $-0,32(-0,25,-0,07)$ & $-0,30(-0,18,-0,12)$ \\
Kohorte vor 1949 geboren & $0,24(0,14,0,10)$ & $0,33(0,07,0,26)$ \\
Bildung Eltern & $-0,16(-,-0,16)$ & $-0,20(-,-0,20)$ \\
Berufstätigkeit Mutter & $-0,10(-0,10,-)$ & n.s. (n.s., n.s. $)$ \\
Religiosität Eltern & $0,04(-, 0,04)$ & $0,06(-, 0,06)$ \\
Wertorientierung & & \\
Antiegalitäre Haltung & $0,30(0,30,-)$ & $0,26(0,26,-)$ \\
$\begin{array}{l}\text { Weitere Sozio-demografische Variab- } \\
\text { len und Konstrukte der Situation }\end{array}$ & $-0,28(-0,13,-0,15)$ & $-0,37(-0,18,-0,19)$ \\
Eigene Bildung & & $-0,22(-0,22,-)$ \\
Eigene Berufstätigkeit (Frauen) & & \\
Berufstätigkeit Partnerin (Männer) & $-0,18(-0,14,-0,04)$ & $0,15(0,19,-0,05)$ \\
Eigene Religiosität & $0,09(0,13,-0,04)$ & $0,33(0,33,-)$ \\
Anomia & $0,21(0,30,-0,09)$ & $0,05(\mathrm{n}, \mathrm{s}, 0,05)$ \\
Wahrnehmung ökonomischer & $0,15(0,13,0,02)$ & \\
Deprivation & & $45 \%$ \\
$\mathrm{R}^{2}$ & $39 \%$ &
\end{tabular}

*Alle aufgeführten Koeffizienten sind mindestens auf dem $5 \%$-Niveau signifikant

aber schwächer ausgeprägt sind die Sozialisationseffekte des Elternhauses. Vor allem das elterliche Bildungsniveau sowie die mütterliche Berufstätigkeit sind hier erwähnenswert. Eine insgesamt nur geringe Bedeutung für das Geschlechtsrollenverständnis der Männer besitzt die Religiosität und zwar sowohl die eigene religiöse Bindung als auch jene der Eltern.

Vergleicht man auch für Frauen die totalen Effektgrößen der verschiedenen Konstrukte miteinander, so fällt auf, dass der insgesamt stärkste Einfluss auf die Geschlechtsrollenorientierungen vom Faktor Bildung ausgeht (totaler Effekt $=-0,37$ ). Der Effekt der Schulbildung verläuft jeweils zur Hälfte direkt und indirekt (Tab.2): Indirekt reduziert ein höheres Bildungsniveau sexistische Einstellungen, indem es antiegalitäre Haltungen befördert, bei Frauen zu vermehrter Berufstätigkeit führt und Gefühle von Anomia verringert. ${ }^{20}$ Ferner besteht zusätzlich eine substanzielle direkte Wirkung der Bildung auf Geschlechtsrollenvorstellungen. Über die sich hinter diesem Effekt verbergenden Ursachen lassen sich einige Vermutungen anstellen. So ist zu erwarten, dass höher gebildete Frauen zum einen eher in der Lage sind, die (für sie meist negativen) Konsequenzen traditioneller Geschlechtsrollenverhältnisse abzuschätzen. Zum anderen verfügen gebildete Frauen in der Regel über höhere berufliche Aspirationen und auch über die Fähigkeiten,

20 Diese Wirkmechanismen gelten im Prinzip auch für Männer, wenngleich auch in etwas abgeschwächter Form. 
diese umzusetzen. Dass sich die Attraktivität traditioneller Geschlechtsrollen für höher gebildete Frauen in Grenzen hält, ist daher durchaus plausibel.

Aber auch Anomia als Effekt der wahrgenommenen Situation (totaler Effekt =0,33), die zum größeren Teil indirekt wirksame Kohortenzugehörigkeit (totaler Effekt =0,33) sowie der Ort der Sozialisation (Ost- vs. Westdeutschland, totaler Effekt $=-0,30$ ) verfügen über eine erhebliche Wirkung auf die Geschlechtsrollenauffassungen von Frauen. Etwas weniger einflussreich zeigen sich dagegen die Faktoren antiegalitäre Haltung, eigene Berufstätigkeit, elterliche Bildung sowie eigene Religiosität. Als nahezu oder gänzlich bedeutungslos für sexistische Einstellungen bei Frauen stellen sich schließlich das Empfinden ökonomischer Benachteiligung, Berufstätigkeit der Mutter sowie die religiöse Bindung im Elternhaus heraus (Tab. 2).

Vergleicht man darüber hinaus die Wirkmechanismen der Erklärungsfaktoren zwischen den Geschlechtern miteinander ${ }^{21}$, so fällt auf, dass bei Männern die Kohortenzugehörigkeit stärker direkt (Abb.6) als vermittelt (Tab.2) wirkt. Bei Frauen dagegen verläuft der Kohorteneffekt zum überwiegenden Teil indirekt (Tab.2). Die indirekten Einflusspfade bei Frauen bedeuten konkret, dass ältere Frauen vor allem deshalb über sexistischere Einstellungen verfügen, weil sie weniger Bildung genossen haben und seltener einer Berufstätigkeit nachgehen oder nachgegangen sind als jüngere Frauen. Überdies sind die vor 1949 geborenen Frauen stärker an Religionen sowie an antiegalitäre Werthaltungen gebunden, was traditionelle Rollenorientierungen begünstigt. Der verbleibende direkte Kohorteneffekt, der nicht über diese Mediatoren verläuft, ist bei Frauen mit einem BetaWert von 0,07 nur sehr schwach ausgeprägt (s. Abb.7). Bei Männern liegt der direkte Kohorteneinfluss mit einem Beta-Wert von 0,14 etwas höher. ${ }^{22}$ Man könnte diesen Befund tendenziell so deuten, dass Männer eher den Rollenauffassungen anhängen, die sie früh gelernt haben. ${ }^{23}$ Für Frauen spielen dagegen intervenierende Größen wie Bildung eine etwas gewichtigere Rolle für die Entwicklung der Geschlechtsrollenvorstellungen. Das Ergebnis, dass der Bildungseffekt bei Frauen stärker ausgeprägt ist, lässt sich möglicherweise damit erklären, dass Bildung im Hinblick auf die theoretischen Modelle zweifach wirkt: Zum einen über die Sozialisationswirkung der Bildungsinstitutionen (exposurebased-Modell), zum anderen aber auch darüber, dass höher gebildete Frauen anders als höher gebildete Männer infolge ihrer beruflichen Aspirationen und Kompetenzen ein stärkeres Interesse an egalitären Geschlechtsrollen entwickeln (interest-based-Modell).

Betrachtet man auch den Einfluss der Herkunft (Sozialisation in Ost- oder Westdeutschland) im Detail, so stellt sich heraus, dass bei beiden Geschlechtern der indirekt

21 Ein direkter, statistisch abgesicherter Vergleich der Effektstärken zwischen Männern und Frauen ist hier nicht möglich, da aufgrund der unterschiedlichen Variablen (eigene Berufstätigkeit bei Frauen oder Berufstätigkeit der Partnerin bei Männern) kein Gruppenvergleich berechnet werden konnte. Bei einem Vergleich der Geschlechter müssen zudem die unstandardisierten BetaKoeffizienten betrachtet werden (nicht ausgewiesen). Auch wenn ein statistisch abgesicherter Vergleich zwischen Männern und Frauen hier nicht möglich ist, so können doch die beiden Teilstichproben der Männer und Frauen deskriptiv miteinander verglichen werden.

22 Die indirekten Pfade bei Männern zwischen Kohortenzugehörigkeit und Sexismus ähneln denen der Frauen, sind aber etwas schwächer ausgeprägt.

23 Es könnte allerdings auch sein, dass sich der direkte Effekt weiter reduziert, wenn dritte, hier nicht berücksichtigte Variablen bei Männern von Bedeutung sind. 
verlaufende Effekt überraschenderweise geringer ist als die direkte Wirkung (Tab. 2 und Abb. 6 und 7). Indirekt reduziert die Variable Sozialisationsort sexistische Einstellungen insbesondere über die häufigere Berufstätigkeit ostdeutscher Frauen oder Mütter sowie über die ausgeprägteren antiegalitären Werthaltungen in diesen Regionen. Es verbleibt ein deutlicher direkter Effekt dieses Faktors (bei Männern =0,25, bei Frauen 0,18, Abb. 6 und 7), der auf weitere Ost-West-Unterschiede hinweist. Als mögliche Ursache kommen hier mehrere Faktoren in Frage. Zum einen ist denkbar, dass frühe ideologische Prägungen durch die beiden Gesellschaftssysteme, die in den vorliegenden Modellen nicht unmittelbar berücksichtigt werden konnten, für die verschiedenen Geschlechtsrollenvorstellungen mitverantwortlich sind. Zum anderen könnten die unterschiedlichen Lebensbedingungen in Ost- und Westdeutschland heute zu den Differenzen in den Rollenauffassungen beitragen. ${ }^{24}$ So ist beispielsweise vorstellbar, dass im Osten sexistische Einstellungen auch deshalb seltener anzutreffen sind, weil die Menschen hier im Vergleich zu den westdeutschen Landesteilen und unabhängig von den sozio-ökonomischen Verhältnissen es eher für notwendig halten, dass beide Partner zum Haushaltseinkommen beitragen (vgl. Braun et al. 1994).

\section{Zusammenfassung und Diskussion}

Fasst man die wesentlichen Befunde der vorliegenden Untersuchung zusammen, so lassen sich einige grundsätzliche Ergebnisse festhalten.

Traditionell-sexistische Einstellungen sind nach wie vor bei einem relevanten Teil der Bevölkerung anzutreffen. Im Hinblick auf egalitäre Geschlechtsrollenvorstellungen unterentwickelt sind vor allem die westlichen Landesteile.

Insgesamt bestätigen Analysen zum großen Teil die theoretischen Erwartungen. Für Männer konnten alle Hypothesen bestätigt werden, für Frauen trifft dies in zwei Fällen nicht zu: Mütterliche Erwerbstätigkeit führt bei Frauen nicht zur Verringerung sexistischer Einstellungen (Hypothese H5). Und ebenfalls ohne Bedeutung für die Geschlechtsrollenorientierung ist bei Frauen die Perzeption ökonomischer Deprivation (Hypothese H10).

Für sexistische Einstellungen sind sowohl Effekte der Sozialisation, inklusive der Wertorientierungen, als auch Einflüsse der Situation von Bedeutung. Die Effekte der Sozialisation verlaufen nur zum Teil ausschließlich indirekt (Religiosität und Bildung des Elternhauses). Der andere Teil der Sozialisationsvariablen (Berufstätigkeit Mutter, Kohortenzugehörigkeit, Sozialisation im Osten) wirkt sowohl indirekt als auch zusätzlich direkt auf die Geschlechtsrollenvorstellungen. Bei den indirekt verlaufenden Effekten sind Bildung, antiegalitäre Wertorientierungen und Anomia die wichtigsten Mediatoren.

$24 \mathrm{Da}$ ein Großteil der in Ostdeutschland sozialisierten Personen auch nach wie vor in den östlichen Landesteilen wohnt, misst die die Variable „Sozialisation in Ostdeutschland“ nicht nur den Effekt der Sozialisation, sondern auch Unterschiede zwischen Ost- und Westdeutschland zum aktuellen Zeitpunkt. Diese Unterschiede sind allerdings schon zu einem gewissen Anteil kontrolliert, etwa durch das Konstrukt „ökonomische Deprivation“. 
Im Wesentlichen direkt wirken hingegen die Effekte der wahrgenommenen Situation (Anomia und Ökonomische Deprivation).

Die insgesamt wichtigsten Determinanten zur Erklärung sexistischer Einstellungen sind (mangelnde) Bildung, westdeutsche Herkunft, die Zugehörigkeit zur Kohorte der vor 1949 Geborenen, antiegalitäre Werthaltungen sowie anomische Empfindungen. Relativ schwach ausgeprägt ist der Effekt der Religiosität, wenngleich er, wie erwartet, einen Anstieg sexistischer Einstellungen bewirkt. Die Tatsache, dass die Wirkung der Religiosität nur schwach ausgebildet ist, könnte jedoch eine Folge der Operationalisierung sein, die ausschließlich über die Variable Kirchgangshäufigkeit erfolgte.

Bei der Gewichtung der verschiedenen Erklärungsgrößen treten einige nennenswerte Unterschiede zwischen Männern und Frauen zutage:25

- Der Faktor Alter, in Form der Kohortenzugehörigkeit, spielt bei Frauen eine etwas gewichtigere Rolle bei der Erklärung von sexistischen Einstellungen als bei Männern. Dieser Befund geht vor allem auf den deutlichen indirekten Kohorteneffekt bei Frauen zurück. D.h. der Effekt lässt sich insbesondere damit erklären, dass ältere Frauen weniger Bildung genossen haben und seltener berufstätig sind oder waren.

- Besonders bei Frauen reduziert ein hohes Bildungsniveau mittelbar und unmittelbar traditionelle Geschlechtsrollenvorstellungen. Der stärkere Bildungseffekt für Frauen deutet darauf hin, dass bei Frauen zusätzlich zur Sozialisationswirkung der Bildungsinstitutionen auch die Interessenlage infolge der höheren Bildung emanzipierte Geschlechtsrollenvorstellungen begünstigt.

- Desintegrationserscheinungen in Gestalt von Anomia begünstigen bei beiden Geschlechtern traditionelle Rollenauffassungen. Bei Frauen ist dieser Effekt allerdings etwas stärker ausgeprägt als bei Männern.

Die Effekte von Anomia und bei Männern der Wahrnehmung ökonomischer Deprivation weisen insgesamt auf die Bedeutung von Emotionen für die Ausbildung von Geschlechtsrollenorientierungen hin (vgl. auch Rippl und Baier 2005). Die empirischen Befunde sprechen dafür, dass sexistische Einstellungen tatsächlich eine Verarbeitungsstrategie für Deprivationsgefühle und anomische Empfindungen darstellt und den Wunsch nach klaren Strukturen, Ordnung und Sicherheit befriedigt. Über die Frage, warum die Wahrnehmung ökonomischer Deprivation lediglich bei Männern Wirkung zeigt, können nur Mutmaßungen angestellt werden. Möglicherweise beruht das Ergebnis darauf, dass Männer einen größeren ökonomischen Druck verspüren, weil sie sich stärker als Frauen für die ökonomische Versorgung der Familie verantwortlich fühlen.

Dass Anomia Einfluss auf politische Orientierungen besitzt, konnte auch an anderer Stelle gezeigt werden (Kühnel et al. 2009). Geht man davon aus, dass anomische Gefühle, d. h. pessimistische Zukunftsprognosen und der Eindruck, dass die Politik keine adäquaten Problemlösungen bereitstellt, in Zeiten globaler Wirtschaftskrisen verstärkt werden, dann ist zu erwarten, dass auch sexistische Positionen weiter Zulauf erhalten. Ähnliches gilt, zumindest bei Männern, für das Gefühl ökonomischer Benachteiligung. Da die negativen Folgen der Wirtschaftskrise insbesondere die sozio-ökonomisch Benachteiligten

25 Auch wenn ein statistisch abgesicherter Vergleich zwischen Männern und Frauen hier nicht möglich ist (vgl. Fußnote 16), so können doch die beiden Teilstichproben der Männer und Frauen deskriptiv miteinander verglichen werden. 
trifft (Arbeitslosigkeit, Abbau von staatlichen Transferleistungen etc.), ist zu befürchten, dass die betroffenen Männer auch hier ihre sexistischen Vorstellungen weiter ausbauen.

Die Frage, ob sich die Geschlechtsrollenvorstellungen in Ost- und Westdeutschland mittelfristig angleichen oder ob sie im Gegenteil weiter auseinander driften, ist nicht leicht zu beantworten. So sind einerseits in den ostdeutschen Bundesländern egalitäre Wertvorstellungen auch heute noch, zwanzig Jahre nach der Wiedervereinigung, sehr viel weiter verbreitet als in Westdeutschland; auch wenn die langfristig wirksamen gesellschaftspolitischen Prägungen der DDR mit der Zeit insgesamt etwas verblassen dürften. Bleiben diese Wertunterschiede zwischen Ost- und Westdeutschland bestehen, dann ist auch weiterhin mit tendenziell unterschiedlichen Geschlechtsrollenauffassungen zu rechnen (vgl. Lee et al. 2007).

Berücksichtigt man andererseits das höhere Niveau gesellschaftlicher Desintegrationserscheinungen (Anomia) und die nach wie vor ungünstige ökonomische Situation in Ostdeutschland, die zumindest für Männer eine gewisse Rolle spielt, so ist zu erwarten, dass traditionell-sexistische Rollenvorstellungen in Ostdeutschland eher befördert werden.

Sollte das Bildungsniveau der Bevölkerung allerdings weiter ansteigen, dann ist hingegen zu erwarten, dass sexistische Einstellungen in Zukunft insgesamt weiter an Bedeutung verlieren. Somit unterstreichen die vorliegenden Befunde die herausragende Bedeutung des Faktors Bildung (vgl. auch Rippl 2002; Heyder 2003). Will man also die im Grundgesetz verankerte Gleichstellung der Geschlechter voranbringen und sexistische Einstellungen aus den Köpfen der Menschen verbannen oder gar nicht erst entstehen lassen, so ist es unabdingbar, mehr in Bildung $\mathrm{zu}$ investieren. Und es sollten, vom normativen Standpunkt aus betrachtet, egalitäre Werthaltungen gefördert werden, da sie eine merkliche Reduktion sexistischer Einstellungen bewirken. Wenn sich zudem die Bedingungen für die Erwerbstätigkeit von Frauen auf dem Arbeitsmarkt künftig verbessern, dann ist zu erwarten, dass sich egalitärere Geschlechtsrollenorientierungen weiter ausbreiten.

$\mathrm{Ob}$ es zu einer langfristigen Veränderung der Geschlechtsrollenorientierungen kommt, und wie sich die anderen diskutierten (oder weitere im vorliegenden Datensatz nicht enthaltene) Einflussgrößen auswirken, sollte durch künftige Untersuchungen untersucht werden.

Abschließend ist darauf hinzuweisen, dass die hier vorgestellten Resultate in Ermangelung geeigneter Längsschnittdaten auf einer Analyse mit Querschnittdaten beruhen. Da die Kausalrichtung der Variablen letztlich nur mithilfe von Längsschnittdaten geklärt werden kann, wäre es für die Zukunft wünschenswert, Items zu Geschlechtsrollenorientierungen in Paneluntersuchungen mit aufzunehmen.

Danksagung: Für wertvolle Kommentare danke ich den Herausgebern und den Gutachtern der KZfSS sowie Andreas Günther, Steffen Kühnel und Manfred Mays. 


\section{Anhang}

Tab. 3: Itemformulierungen

\begin{tabular}{ll}
\hline Konstrukt & Itemformulierung \\
\hline Ökonomische Deprivation & \\
F002 & Und Ihre eigene wirtschaftliche Lage heute? \\
& 1 Sehr gut, 2 Gut, 3 Teils gut teils schlecht,4 Schlecht, 5 Sehr \\
& schlecht \\
& Im Vergleich dazu, wie andere hier in Deutschland leben: \\
& Glauben Sie, dass Sie Ihren gerechten Anteil erhalten, mehr \\
& als Ihren gerechten Anteil, etwas weniger oder sehr viel \\
& weniger? \\
& 1=mehr als den gerechten Anteil, 2=den gerechten Anteil, \\
& 3=etwas weniger als den gerechten Anteil, 4= sehr viel weni- \\
& ger als den gerechten Anteil
\end{tabular}

Anomia

F035_2

F035_3

Antiegalitarismus

F024_B

F024_C

Traditioneller Sexismus

F032_B

F032_D

F032_F
So wie die Zukunft aussieht, kann man es kaum noch verantworten, Kinder auf die Welt zu bringen

Die meisten Politiker interessieren sich in Wirklichkeit gar nicht für die Probleme der einfachen Leute

$1=$ bin anderer Meinung, $2=$ bin derselben Meinung

Die Rangunterschiede zwischen den Menschen sind akzeptabel, weil sie im Wesentlichen ausdrücken, was man aus den Chancen, die man hatte, gemacht hat

Ich finde die sozialen Unterschiede in unserem Land im Großen und Ganzen gerecht

$1=$ Stimme überhaupt nicht $z u, 2$ =Stimme eher nicht $z u$,

$3=$ Stimme eher $z u, 4=$ Stimme voll $z u$

Für eine Frau ist es wichtiger, ihrem Mann bei seiner Karriere zu helfen, als selbst Karriere zu machen

Es ist für alle Beteiligen viel besser, wenn der Mann voll im Berufsleben steht und die Frau zu Hause bleibt und sich um den Haushalt und die Kinder kümmert.

Eine verheiratete Frau sollte auf eine Berufstätigkeit verzichten, wenn es nur eine begrenzte Anzahl von Arbeitsplätzen gibt, und wenn ihr Mann in der Lage ist, für den Unterhalt der Familie zu sorgen

$1=$ Stimme überhaupt nicht $z u, 2=$ Stimme eher nicht $z u$, $3=$ Stimme eher $z u, 4=$ Stimme voll und ganz zu 
Tab. 4: Messmodell, standardisierte Indikatorenladungen und Residualvarianzen.* (Quelle: ALLBUS 2008, eigene Berechnungen)

\begin{tabular}{|c|c|c|c|c|}
\hline \multirow[t]{2}{*}{ Konstrukt } & \multicolumn{2}{|l|}{ Männer } & \multicolumn{2}{|l|}{ Frauen } \\
\hline & $\begin{array}{l}\text { Indikatoren- } \\
\text { ladungen }\end{array}$ & $\begin{array}{l}\text { Residual- } \\
\text { varianzen }\end{array}$ & $\begin{array}{l}\text { Indikatoren- } \\
\text { ladungen }\end{array}$ & $\begin{array}{l}\text { Residual- } \\
\text { varianzen }\end{array}$ \\
\hline \multicolumn{5}{|l|}{ Religiosität Eltern: } \\
\hline Religiosität Mutter & 0,93 & 0,14 & 0,92 & 0,16 \\
\hline Religiosität Vater & 0,91 & 0,18 & 0,85 & 0,28 \\
\hline \multicolumn{5}{|l|}{ Bildung Eltern } \\
\hline Bildung Mutter & 0,79 & 0,38 & 0,80 & 0,35 \\
\hline Bildung Vater & 0,87 & 0,28 & 0,81 & 0,35 \\
\hline \multicolumn{5}{|l|}{$\begin{array}{l}\text { Wahrnehmung ökonomischer } \\
\text { Deprivation }\end{array}$} \\
\hline Bewertung eigene wirtschaftliche Lage & 0,73 & 0,55 & 0,66 & 0,56 \\
\hline $\begin{array}{l}\text { Gerechter Anteil am Lebensstandard } \\
\text { Anomia }\end{array}$ & 0,70 & 0,51 & 0,63 & 0,52 \\
\hline Bei dieser Zukunft keine Kinder mehr & 0,59 & 0,65 & 0,59 & 0,65 \\
\hline $\begin{array}{l}\text { Politiker uninteressiert an einfachen } \\
\text { Leuten }\end{array}$ & 0,52 & 0,73 & 0,50 & 0,75 \\
\hline \multicolumn{5}{|l|}{ Antiegalitäre Haltung } \\
\hline Rangunterschiede sind akzeptabel & 0,67 & 0,59 & 0,74 & 0,50 \\
\hline Soziale Unterschiede sind gerecht & 0,77 & 0,41 & 0,73 & 0,47 \\
\hline \multicolumn{5}{|l|}{ Sexismus } \\
\hline $\begin{array}{l}\text { Frau soll lieber Mann bei der Karriere } \\
\text { helfen }\end{array}$ & 0,64 & 0,59 & 0,69 & 0,52 \\
\hline $\begin{array}{l}\text { Frau soll zu Hause bleiben und Kinder } \\
\text { versorgen }\end{array}$ & 0,84 & 0,30 & 0,83 & 0,30 \\
\hline $\begin{array}{l}\text { Frau soll nach Heirat Arbeitsplatz } \\
\text { freimachen }\end{array}$ & 0,69 & 0,53 & 0,72 & 0,48 \\
\hline \multicolumn{5}{|l|}{ Nebenladungen: } \\
\hline Religion Eltern - Bildung Mutter & - & & $-8,08$ & \\
\hline $\begin{array}{l}\text { Anomia - Rangunterschiede sind } \\
\text { akzeptabel }\end{array}$ & 0,19 & & 0,22 & \\
\hline Kohorte vor 1949 - Bildung Vater & 0,10 & & - & \\
\hline $\begin{array}{l}\text { Kohorte vor } 1949 \text { - Rangunterschiede } \\
\text { sind akzeptabel }\end{array}$ & 0,11 & & 0,09 & \\
\hline $\begin{array}{l}\text { Sozialisation in Ostdeutschland - } \\
\text { Bewertung eigene wirtschaftliche Lage }\end{array}$ & 0,19 & & - & \\
\hline $\begin{array}{l}\text { Sozialisation in Ostdeutschland - } \\
\text { Gerechter Anteil am Lebensstandard }\end{array}$ & - & & 0,20 & \\
\hline
\end{tabular}


Open Access: Dieser Artikel unterliegt den Bedingungen der Creative Commons Attribution License. Dadurch sind die Nutzung, Verteilung und Reproduktion erlaubt, sofern der/die Originalautor/en und die Quelle angegeben sind.

\section{Literatur}

Arbuckle, James L. 1996. Full information estimation in the presence of incomplete data.In Advanced structural equation modeling: Issues and techniques, Hrsg. George A. Marcoulides und 1. E Randel Schumacker, 243-277. Hillsdale: Erlbaum.

Banaszak, Lee Ann, und Eric Plutzer. 1993. Contextual determinants of feminist attitudes: National and subnational influences in Western Europe. American Political Science Review 87:147-157.

Bollen, Kenneth. A. 1989. Structural equations with latent variables. New York: Wiley.

Bolzendahl, Catherine, und Daniel J. Myers. 2004. Feminist attitudes and support for gender equality: Opinion change in women and men, 1974-1998. Social Forces 83:759-789.

Braun, Michael, Duane Alwin und Jacqueline Scott. 1994. Wandel der Einstellungen zur Rolle der Frau in Deutschland und den Vereinigten Staaten. In Blickpunkt Gesellschaft 3, Hrsg. Michael Braun und Peter Mohler, 151-173. Opladen: Westdeutscher.

Brewster, Karin. L., und Irene Padavic. 2000. Change in gender-ideology, 1977-1996: The contributions of intra cohort change and population turnover. Journal of Marriage and the Family 62:477-487.

Bryant, Alyssa N. 2003. Changes in attitudes toward women's roles: Predicting gender-role traditionalism among college students. Sex Roles 48:131-142.

Davis, Nancy J., und Robert V. Robinson. 1991. Men's and women's consciousness of gender inequality: Austria, West Germany, Great Britain, and the United States. American Sociological Review 56:72-84.

Davis, Shannon N., und Theodore N. Greenstein. 2009. Gender Ideology: Components, predictors, and consequences. Annual Review of Sociology 35:87-105.

Eckes, Thomas. 2008. Geschlechterstereotype: Von Rollen, Identitäten und Vorurteilen. In Handbuch Frauen- und Geschlechterforschung: Theorie, Methode, Empirie, Hrsg. Ruth Becker und Barbara Budrich, 171-182. Wiesbaden: VS Verlag für Sozialwissenschaften.

Eckes, Thomas, und Iris Six-Materna. 1998. Leugnung von Diskriminierung: Eine Skala zur Erfassung des modernen Sexismus. Zeitschrift für Sozialpsychologie 29:224-238.

Endrikat, Kirsten. 2003. Ganz normaler Sexismus. Reizende Einschnürung in ein Rollenkorsett. In Deutsche Zustände, Folge 2, Hrsg. Wilhelm Heitmeyer, 120-140. Frankfurt a. M.: Suhrkamp.

Festinger, Leon. 1957. A theory of cognitive dissonance. Stanford: Stanford University Press.

Glick, Peter, Maria Lameiras und Yolanda. R. Castro. 2002. Education and Catholic religiosity as predictors of hostile and benevolent sexism toward women and men. Sex Roles 47:433-441.

Hadjar, Andreas. 2006. Bildungsexpansion und Wandel von sozialen Werten. In Die Bildungsexpansion. Erwartete und unerwartete Folgen, Hrsg. Andreas Hadjar und Rolf Becker, 205-230. Wiesbaden: Verlag für Sozialwissenschaften.

Hadjar, Andreas. 2008. Meritokratie als Legitimationsprinzip. Die Entwicklung der Akzeptanz sozialer Ungleichheit im Zuge der Bildungsexpansion. Wiesbaden: VS Verlag für Sozialwissenschaften.

Heyder, Aribert. 2003. Bessere Bildung, bessere Menschen? Genaueres Hinsehen hilft weiter. In Deutsche Zustände. Folge II, Hrsg. Wilhelm Heitmeyer, 78-99. Frankfurt a. M.: Suhrkamp.

Hofäcker, Dirk, und Detlev Lück. 2004. Zustimmung zu traditionellem Alleinverdiener-Modell auf dem Rückzug, Einstellung von Frauen zur geschlechterspezifischen Arbeitsteilung in internationalen Vergleich. Informationsdienst Soziale Indikatoren 32:12-15. 
Hopf, Wulf. 1999. Ungleichheit der Bildung und Ethnozentrismus. Zeitschrift für Pädagogik 45:847-865.

Lee, Kristen S., Duane F. Alwin und Paula A. Tufis. 2007. Beliefs about women's labour in the reunified Germany, 1991-2004. European Sociological Review 23:487-503.

Kühnel, Steffen, Anja Mays und Esther Ochoa Fernández. 2009. Beeinflusst Anomia politische Orientierungen? In Wähler in Deutschland, Sozialer und politischer Wandel, Gender und Wahlverhalten. Hrsg. Steffen Kühnel, Oskar Niedermayer und Bettina Westle, 68-81. Wiesbaden: VS Verlag für Sozialwissenschaften.

Kurz, Karin. 2004. Geschlechterrollen - Und sie bewegen sich doch?! In Strukturierung von Wissen und die symbolische Ordnung der Geschlechter Gender-Tagung Bamberg 2003, Hrsg. Marianne Heimbach-Steins, Bärbel Kerkhoff-Hader, Elenore Ploil und Ines Weinrich, 83-89. Münster: Lit-Verlag.

LBS-Kinderbarometer Deutschland. 2009. Stimmungen, Trends und Meinungen von Kindern aus Deutschland. Ergebnisse des Erhebungsjahres 2008/09. http://www.lbs.de/bw/lbs/pics/upload/ tfmedia1/HBNAAyMa4vT.pdf. (Zugegriffen: 18. Okt. 2010).

Mays, Anja. 2009. Einfluss jugendlicher Sozialisationserfahrungen auf ausgewählte Aspekte der politischen Identität im Erwachsenenalter. http://webdoc.sub.gwdg.de/diss/2009/mays/mays. pdf. (Zugegriffen: 1. Okt. 2010).

Morgan, Carolyn S., und Alexis J. Walker. 1983. Predicting sex role attitudes. Social Psychology Quarterly 46:148-151.

Plutzer, Eric. 1991. Preferences in family politics: Women's consciousness or family context? Political Geography Quarterly 10:162-173.

Reinecke, Jost. 2005. Strukturgleichungsmodelle in den Sozialwissenschaften. München: Oldenbourg

Rhodebeck, Laurie A. 1996. The structure of men's and women's feminist orientations, feminist identity and feminist opinion. Gender and Society 10:386-403.

Rippl, Susanne. 2002. Bildung und Fremdenfeindlichkeit. Die Rolle schulischer und familialer Sozialisation zur Erklärung von Bildungsunterschieden im Ausmaß von fremdenfeindlichen Einstellungen. Kölner Zeitschrift für Soziologie und Sozialpsychologie 54:135-146.

Rippl Susanne, und Dirk Baier. 2005. Das Deprivationskonzept in der Rechtsextremismusforschung. Kölner Zeitschrift für Soziologie und Sozialpsychologie 57:644-666.

Räthzel, Nora. 2004. Rassismustheorien: Geschlechterverhältnisse und Feminismus. In Handbuch Frauen- und Geschlechterforschung. Theorie, Methoden, Empirie. Hrsg. Ruth Becker und Beate Kortendieck, 249-256. Wiesbaden: VS Verlag für Sozialwissenschaften.

Sidanius, James, und Felicia Pratto. 1999. Social dominance: An intergroup theory of social hierarchy and oppression. New York: Cambridge University Press.

Thornton, Arland, Duane F. Alwin und Donald Camburn. 1983. Causes and consequences of sexroles attitudes and attitude change. American Sociological Review 48:211-227.

Tomeh, Aida K. 1979. Sex role orientation and structural correlates. The Sociological Quarterly 20:333-344.

Vorwerk Familienstudie. 2009. Institut für Demoskopie Allensbach. http://corporate.vorwerk. com/fileadmin/data/pdf/Publikationen/vorwerk_familienstudie2009.pdf. (Zugegriffen: 18. Okt. 2010).

Westle, Bettina. 2009. Immer noch in der Steinzeit? Gesellschaftliche und politische Gender-Orientierungen. In Wähler in Deutschland. Sozialer und politischer Wandel, Gender und Wahlverhalten, Hrsg. Steffen Kühnel, Oskar Niedermayer und Bettina Westle, 137-165. Wiesbaden: VS Verlag für Sozialwissenschaften. 
World Economic Forum 2010. The Global Gender Gap Report 2010. http:/www3.weforum.org/ docs/WEF_GenderGap_Report_2010.pdf. (Zugegriffen: 19. Dez. 2010).

Anja Mays, 1969, Dr., wissenschaftliche Mitarbeiterin, Methodenzentrum Sozialwissenschaften, Georg-August-Universität Göttingen. Forschungsgebiete: Politische Sozialisation, Analyse politischer Einstellungen und politischen Verhaltens, empirische Wahlforschung. Veröffentlichungen: Beeinflusst Anomia politische Orientierungen? In: Wähler in Deutschland. Sozialer und politischer Wandel, Gender und Wahlverhalten (Hrsg. St. Kühnel et al.), Wiesbaden 2009 (mit S. Kühnel, E. Ochoa Fernàndez); Der Einfluss jugendlicher Sozialisationserfahrungen auf ausgewählte Aspekte der politischen Identität im Erwachsenenalter, Göttingen 2009. 